\title{
Una aproximación conceptual a la retención estudiantil en Latinoamérica ${ }^{1}$
}

\author{
Alexandra Pedraza Ortiz², \\ Vianney Rocío Díaz Pérez ${ }^{3}$, \\ Omar Cabrales Salazar
}

Fecha de recepción: 12 de agosto de 2014

Fecha de revisión: 25 de septiembre de 2014

Fecha de aprobación: 30 octubre de 2014

\section{Resumen}

El presente artículo expone, en primera instancia, un panorama general del estado actual de la educación superior y la deserción estudiantil en Latinoamérica. Para el análisis, se pone en evidencia que el concepto de deserción tiene múltiples facetas, aunque son varios los autores que coinciden en que es un abandono no forzoso de las aulas de clase. Finaliza con sugerencias puntuales para disminuir el abandono de las aulas por parte de los estudiantes.

1 El presente artículo es producto de la investigación titulada "Retención estudiantil en las maestrías del área de educación de Colombia: estrategias académicas", inscrita en el grupo de investigación "Cultura de Desarrollo Humano", adelantada por los autores de este artículo y financiada por la Vicerrectoría de Investigaciones de la Universidad Militar Nueva Granada, Colombia.

2 Co-investigadora. Magíster en educación. Directora de Pregrados y profesora de tiempo completo de la Facultad de Educación y Humanidades, Universidad Militar Nueva Granada, Colombia. Correo electrónico: alexandra.pedraza@unimilitar.edu.co

3 Investigadora principal. Magíster en Educación. Coordinadora de la Maestría en Educación y profesora de tiempo completo de la Facultad de Educación y Humanidades, Universidad Militar Nueva Granada, Colombia. Correo electrónico: vianney.diaz@unimilitar.edu.co

4 Co-investigador. Magíster en Educación. Director de Humanidades y profesor de tiempo completo de la Facultad de Educación y Humanidades, Universidad Militar Nueva Granada, Colombia. Correo electrónico: omar.cabrales@unimilitar.edu.co 
Palabras clave

Retención estudiantil, deserción estudiantil, educación superior.

\title{
A conceptual approach to student retention in Latin America
}

\begin{abstract}
This article presents, in the first instance, an overview of the current state of higher education and student desertion in Latin America. For the analysis, it shows that the concept of desertion is multifaceted, although several authors agree that it is a non-forced abandonment of the classroom. It concludes with specific suggestions to reduce the abandonment of the classroom by students.
\end{abstract}

\section{Keywords}

Student retention, student desertion, higher education.

\section{Uma abordagem conceitual para a retenção dos alunos na América Latina}

\section{Resumo}

Este artigo apresenta, em primeiro lugar, uma visão geral do estado atual do ensino superior e da evasão escolar na América Latina. Para a análise, isso mostra que o conceito de atrito é multifacetado, apesar de que vários autores concordam que é um abandono não forçado das salas de aula. Termina com sugestões específicas para reduzir o abandono da sala de aula pelos alunos. 


\section{Palavras-Chave}

Retenção dos alunos, evasão escolar, ensino superior.

\section{Introducción}

Con el fin de combatir o disminuir la problemática de la deserción estudiantil en la educación superior, surgen políticas que proponen el diseño y la implementación de programas y estrategias orientados a la persistencia y permanencia de los estudiantes en las aulas.

En vista del limitado estudio que se ha hecho sobre la deserción estudiantil en el campo de la educación posgradual, el aterrizaje de esta temática plantea diversos retos para este nivel formativo en el campo de la educación, entre los que se cuenta promover estrategias de retención de manera que las tasas crecientes -de las cuales dan fe investigaciones recientes- disminuyan. De acuerdo con lo anterior, en la primera parte de este documento se lleva a cabo un diagnóstico de la situación de la educación superior, luego se definen brevemente los conceptos a aplicar y finalmente se sugieren opciones para disminuir la deserción.

\section{Un vistazo al panorama de la educación superior}

De acuerdo con el Ministerio de Educación Nacional (2008), la educación superior podría definirse como el proceso de formación permanente, personal, cultural y social que se fundamenta en una concepción integral de la persona humana, de su dignidad, de sus derechos y de sus deberes.

Así, la universidad no puede verse solo como un escenario de producción científica. De hecho, desde la Ley 30 de 1992 se le vienen otorgando funciones anexas a la docencia, a la investigación y a la proyección social, con el ánimo de fortalecer su rol en el contexto y promover el desarrollo social, económico y científico del país, de modo que se deleguen en ella responsabilidades que la inviten a enfrentar los cambios mundiales de manera crítica, siempre actuando de forma pertinente y eficiente.

Las universidades no han estado ajenas a estos cambios, y hoy se ven inmersas en las profundas y aceleradas mutaciones de una sociedad que les exige 
calidad y cobertura en un contexto neoliberal altamente competitivo, en el cual deben responderle a un creciente número de estudiantes egresados de la educación secundaria, a una sociedad que todavía espera de ella grandes logros como templo del saber y la investigación, y a un sector empresarial cada vez más exigente, más cambiante y con cada vez menos posibilidades de ofrecerle trabajo digno a todos los egresados.

En atención, principalmente, a su función de docencia y en pos de la formación integral, las universidades día a día acogen un mayor número de estudiantes, tendencia que se puede pensar que viene fortalecida por las acciones de política en tanto cobertura y que le apuntan al mejoramiento de la calidad de la educación superior, al punto de que estudiosos e investigadores (Zabalza, 2007; Sisto 2005; Cabrales, 2008) hablan de la masificación y la universalización de la educación superior.

Tabla 1. Número de matriculados por país, 1975 -2004.

\begin{tabular}{l|l|l|l}
\hline & 1975 & 1995 & 2004 \\
\hline Argentina & 579.736 & 1.054 .145 & 2.026 .735 \\
\hline Bolivia & 49.850 & 154.040 & 346.056 \\
\hline Brasil & 1.089 .808 & 1.661 .034 & 4.163 .733 \\
\hline Chile & 149.647 & 327.074 & 567.114 \\
\hline Colombia & 176.098 & 576.540 & 1.112 .574 \\
\hline Ecuata Rica & 33.239 & 83.608 & 175.284 \\
\hline Mexico & 170.173 & 174.584 & 295.608 \\
\hline Panama & 562.056 & 1.420 .461 & 2.236 .791 \\
\hline Peru & 26.289 & 69.528 & 130.026 \\
\hline Republica Dominicana & 1. & 693.264 & 831.345 \\
\hline Uruguay & 32.627 & 127.409 & 293.565 \\
\hline Venezuela & 213.542 & 74.842 & 109.817 \\
\hline España & 540.238 & 597.487 & 1.074 .350 \\
\hline Portugal & 79.702 & 1.222 .089 & 1.839 .903 \\
\hline Australia & 274.738 & 185.762 & 400.831 \\
\hline Canada & 1.079 .690 & 1.916 .801 & 1.192 .570 \\
\hline
\end{tabular}




\begin{tabular}{l|l|l|l}
\hline Korea & 318.683 & 1.691 .429 & 3.555 .115 \\
\hline Gran Bretaña & 732.947 & 1.258 .188 & 2.247 .441 \\
\hline
\end{tabular}

Fuente: The Task Force on Higher Education and Society, The World Bank, 2000, Statistical Appendix; Informes Nacionales (2006); lesalc, Informe sobre la Educación Superior en América Latina y el Caribe 2000-2005 (2006); Unesco, Global Education Digest (2006). Para Corea, MOEHRD - KEDI, Statistical Yearbook of Education (2004). Recuperado de: http://www.oecd.org/dataoecd/13/33/36040477.pdf

En atención, principalmente, a su función de docencia y en pos de la formación integral, las universidades día a día acogen un mayor número de estudiantes, tendencia que se puede pensar que viene fortalecida por las acciones de política en tanto cobertura y que le apuntan al mejoramiento de la calidad de la educación superior, al punto de que estudiosos e investigadores (Zabalza, 2007; Sisto 2005; Cabrales, 2008) hablan de la masificación y la universalización de la educación superior.

Proporcional a este crecimiento, los retos que se le imponen a la universidad aumentan; a pesar del incremento en los índices de matrícula, los porcentajes de deserción en este mismo nivel no ceden. Se puede afirmar que, aún hoy, uno de cada dos estudiantes abandona el sistema.

Esta problemática aqueja también a los países industrializados, casi tanto como a los que están en vías de desarrollo. En investigaciones adelantadas por varias instituciones de educación superior, se han hallado tasas importantes de deserción a nivel de pregrado y posgrado, a tal punto que el abandono de las aulas es considerado como uno de los factores que más repercute en otras problemáticas sociales. Un estudiante que deserta se convierte en un proyecto de vida frustrado; se reduce sustancialmente su autoestima y puede conducirse a otras circunstancias más graves, como a la adicción a sustancias psicoactivas, por ejemplo.

La Comisión Económica para América Latina y el Caribe (Cepal) (2010) señala que en América Latina solo uno de cada diez jóvenes de 25 a 29 años de edad había completado cinco años de educación superior en el 2010 (un leve aumento comparado con el $7 \%$ presentado en el 2000); como se ve, esta es una problemática que debe preocupar a sistemas e instituciones que se dedican a formar seres humanos y a cualificarlos para que mejoren su calidad de vida y la de sus regiones. 
Las diferencias entre países latinos son muy marcadas. Actualmente, Argentina presenta un $23 \%$ de jóvenes que no completaron su educación terciaria y República Dominicana, un 3\%; mientras que en la última década, México elevó en decenas de veces la proporción de jóvenes con educación terciaria completa, frente a Honduras que la disminuyó a la tercera parte durante el período 2000-2010 (Comisión Económica para América Latina y el Caribe [Cepal], 2010).

Según lo señalado por el Consejo Nacional de Educación Superior (CESU) en el documento La voz de las regiones (2013), en Colombia las tasas de deserción representan un $11 \%$ interanual y un $43 \%$ por cohorte acumulada. Dicho panorama refleja con preocupación indicadores que conducen a indagar más sobre este fenómeno que sacude a la educación superior, y que desencadenan contundentes problemas emocionales y motivacionales, además del posible aumento en las tasas de desempleo.

No se puede negar que la mayoría de instituciones de educación superior han hecho un gran esfuerzo por permitir y facilitar el acceso de estudiantes a sus planteles, pero esto no significa que se les estén brindando las mismas opciones para concluir con éxito sus estudios y que el fenómeno de la deserción haya menguado.

\section{Deserción estudiantil: concepto}

El concepto de deserción estudiantil tiene muchas facetas y ha sido objeto de discusión en cuanto a sus implicaciones y factores. Hacia 2003, empezó a ser estudiado luego de varios análisis costo-beneficio de la educación desde una perspectiva económica en el interior de algunas universidades, aunque con varias dificultades, como el limitado referente teórico y la ausencia de sistematización de sus causales. Entre las aproximaciones conceptuales, se encuentra lo señalado por

Tinto (1982) y Giovagnoli (2002) que definen deserción como una situación a la que se enfrenta un estudiante cuando aspira y no logra concluir su proyecto educativo, considerándose como desertor a aquel individuo que siendo estudiante de una institución de educación superior, no presenta actividad académica durante tres semestres académicos consecutivos. (Castaño et al. 2004)

Por su parte, Páramo y Correa (1999) sustentan que la deserción estudiantil no solo debe ser entendida como el abandono definitivo de las aulas de clase, sino como el abandono de la formación académica, independientemente de las 
condiciones y modalidades de presencialidad; es decisión personal del sujeto y no obedece a un retiro académico forzoso.

Los autores Sánchez y Márquez (2012) exaltan los señalamientos de Fishbein y Ajzen (1975) sobre la deserción como el resultado del debilitamiento de las intenciones iniciales del individuo y de su persistencia; aspectos que a su vez están influenciados por el autoconcepto del estudiante. Así como lo expuesto por Attinasi (1986), quien asegura que la decisión de desertar se ve influenciada por las percepciones y el análisis que el individuo hace de su vida universitaria (Sánchez \& Márquez, 2012).

Así mismo, Ethingon (1991) incorpora la teoría de las "conductas de logro", que comprenden atributos como la perseverancia, la elección y el desempeño, y encuentra que el nivel de aspiraciones y las expectativas de éxito del estudiante son elementos fundamentales para explicar la deserción. De acuerdo con estas definiciones y conceptos, la deserción estudiantil tiene ciertos determinantes e implicaciones que difieren en el tipo de abandono y las causas de este fenómeno; claramente, cada perspectiva depende de los entes a partir de los cuales se requiera visualizarlo.

De manera que el Ministerio de Educación Nacional (2009), analiza cada ente y sus implicaciones:

Desde el punto de vista individual, la deserción hace referencia a las metas y propósitos que tienen los estudiantes al incorporarse a una institución de educación superior. Bajo esta perspectiva, un desertor es aquel individuo que no logra la meta deseada, la cual fue el objetivo por la cual la persona ingresó a una determinada institución de educación superior.

En el caso de los funcionarios, la deserción de un individuo representa el fracaso de la institución que falló en lograr lo que originalmente este se había propuesto al ingresar a la educación superior. Entonces, desde la perspectiva institucional, una medida de la deserción es el número de estudiantes que abandonan la institución de educación superior antes de obtener el título correspondiente (Icfes, 2002). Desde la perspectiva estatal, la deserción comprende el abandono por parte del estudiante al sistema educativo en general. En esta definición, si un estudiante se transfiere de una institución a otra, es considerada como movilidad dentro del sistema pero no como el abandono de todo el sistema de educación superior. Así, es posible identificar las diferentes formas de abandono y diseñar distintas políticas públicas para prevenirlo a los niveles institucional y nacional (Fernández 2009). 
Teniendo en cuenta lo anterior, se evidencia que a pesar de las discrepancias en la caracterización de la deserción, son varios los puntos que coinciden en dichas definiciones; por ejemplo, un abandono voluntario, que de acuerdo con Rivera y colaboradores (2005), puede ser explicado por diferentes categorías de variables: socioeconómicas, individuales, institucionales y académicas.

En ese sentido, las diversas categorías o implicaciones que conllevan a la deserción son abordadas por varios autores que las expresan de manera contundente:

Gráfica 1. Determinantes de la deserción estudiantil.

\section{Determinantes de la deserción}

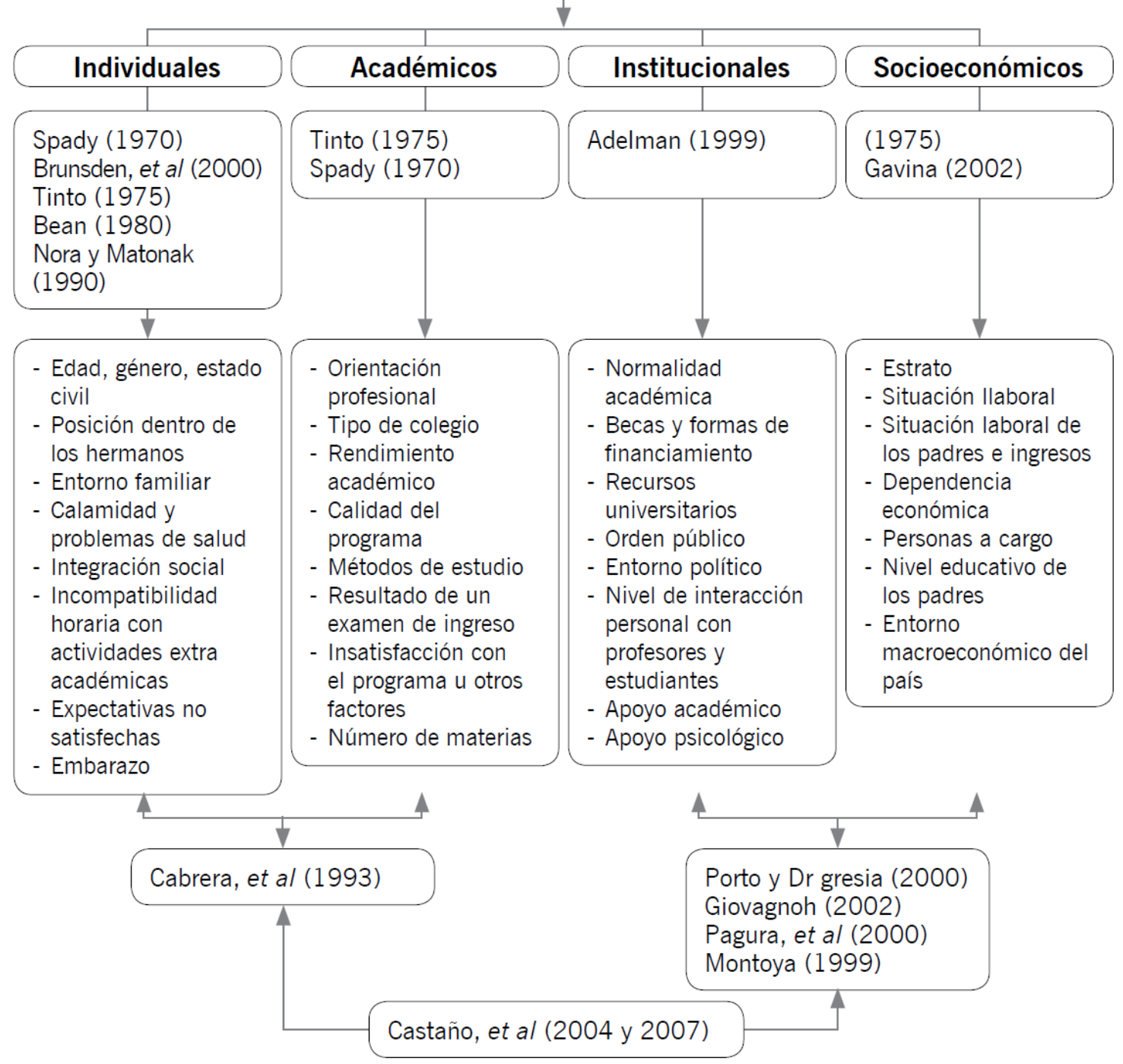

Fuente: Ministerio de Educación Nacional (2008, p. 27). 
Efectivamente, los determinantes que marcan el fenómeno hacen pensar en un amplio abanico de situaciones que llevan al estudiante a dejar sus estudios, y por tanto, implica para las instituciones abordajes diversos que permitan hablar de pertinencia y efectividad en el manejo de la deserción.

\section{Retención estudiantil: un amplio camino por recorrer}

La perspectiva de la deserción hace a las instituciones una invitación que indica su prevención y, por tanto, lleva a pensar en la retención; tal como lo señalan Pineda y Pedraza (2011, p. 21) "son dos fenómenos totalmente entrelazados". Sin embargo, la segunda visualiza un mayor campo de trabajo que genera escenarios para que las instituciones planteen estrategias que, desde cualquier categoría, promuevan la persistencia y permanencia de los estudiantes en las aulas.

La Agencia Interamericana para la Cooperación y el Desarrollo (AICD-OEA) (citado por Pineda y Pedraza, 2011) plantea la retención como "la capacidad que tiene el sistema educativo para lograr la permanencia de los estudiantes en las aulas, garantizando la terminación de ciclos y niveles en los tiempos previstos y asegurando el dominio de las competencias y conocimientos correspondientes" (AICD-OEA, 2006, p. 19). Así las cosas, toda estrategia que pretende promover la retención debe entender al estudiante como "el eje sobre el cual giran todas las acciones propuestas" (AICD-OEA, 2006, p. 158).

En el mismo estudio, Pineda y Pedraza (2011, p. 138) señalan que las universidades han puesto al servicio de sus estudiantes una amplia gama de estrategias (monitorías, tutorías, asesorías, talleres, cursos) que apoyan y promueven, de manera personalizada y grupal, el éxito académico en sus estudiantes al atender sus necesidades particulares haciendo mayor énfasis durante los primeros semestres.

En sintonía con las categorías de deserción expuestas, Swail, Redd y Perna (2003, citado por Pineda y Pedraza, 2011) señalan cinco componentes que constituyen la retención estudiantil: reclutamiento y admisión, servicios académicos, currículo e instrucción, servicios estudiantiles y apoyo financiero. Estos componentes guardan estrecha relación entre ellos, y deben ser monitoreados y mantener comunicación continua que les permita consolidarse como un sistema para el éxito académico.

Entre dichos componentes, los referidos a Servicios Académicos y a Currículo e Instrucción se constituyen en una diada que directamente apoya y facilita el 
desempeño académico de los estudiantes, además de que mejoran los aspectos en los cuales se presentan deficiencias académicas. Así pues, Servicios Académicos está constituido por actividades, programas y planes que implican un acompañamiento al estudiante, con tutorías, mentorías, consejerías, monitorías y cursos especiales en los cuales docentes o pares con mayor recorrido universitario apoyan y orientan al estudiante; además de programas especiales como nivelatorios, vacacionales y remediales.

Por su parte, Currículo e Instrucción está relacionado con la flexibilidad, la movilidad nacional e internacional y las prácticas pedagógicas, que además del conocimiento disciplinar, generan un ambiente académico propicio para el aprendizaje a partir de la relación del profesor y los estudiantes, la cooperación entre los mismos estudiantes, y la dedicación y el compromiso.

El apoyo financiero, factor objeto de múltiples estrategias y programas estatales y del sector privado, pretende amainar las dificultades que, por razones económicas, generan la deserción, de modo que se ofrece al estudiante la posibilidad de adquirir créditos educativos, refinanciaciones de las matrículas o acceder por su rendimiento académico a becas, subsidios o auxilios que mitiguen la carga económica.

De otra parte, la Ley 30 de 1992 en su artículo 117 señala que "las instituciones de educación superior deben adelantar programas de bienestar, entendidos como el conjunto de actividades que se orientan al desarrollo físico, psicoafectivo, espiritual y social de los estudiantes, docentes y personal administrativo". Es en ese marco que los servicios estudiantiles encuentran su nicho y promueven la integración académica, cultural y social del estudiante. Por su parte, las divisiones de reclutamiento y admisión encaminan sus esfuerzos a orientar a los estudiantes a programas que sean coherentes con sus intereses y a facilitar su adaptación al medio universitario.

Este tema y sus categorías han sido objeto de estudio en la formación de pregrado, mientras que los estudios que profundizan esta temática en educación posgradual son limitados, la literatura que circula es escasa y se encuentran aún menos investigaciones. Entre ese escaso avance, está el trabajo de Barrientos y Umaña (2009), titulado Deserción estudiantil en posgrados semipresenciales de la Universidad Estatal a Distancia (UNED), Costa Rica: ¿deserción o retraso?, que en sintonía con la anterior afirmación, ratifican que el estudio de deserción en posgrados es exiguo.

También, se encuentra el trabajo Tesis y deserción: entre el compromiso y el obstáculo, adelantado por Ochoa y Cueva (2012), que refiere a cómo entre los 
estudiantes de la Maestría en Lingüística de la Facultad de Ciencias Humanas de la Universidad Nacional de Colombia, la culminación de sus estudios y en especial la terminación de sus tesis se convierte en el reto de un colectivo, más todavía cuando el promedio de sus tasas de graduación fue de 80\% entre 1998 y 2004 (p. 41).

El 4 de junio de 2010, la Agencia de Noticias de la Universidad Nacional publicó que la deserción en sus posgrados es cercana al 35\% y se concentra principalmente en las maestrías; de igual manera, insiste en la importancia de estudios que profundicen en este fenómeno al nivel de posgrados.

\section{Conclusiones: los retos para la educación posgradual}

Así las cosas, se plantea como un importante desafío la investigación en esta materia, que permita descifrar las estrategias intencionadas e implícitas que ponen en escena los programas posgraduales para mantener a los estudiantes en el aula y, por tanto, cualificar su formación en pos del desarrollo personal y profesional.

Hallar las estrategias que de manera efectiva faciliten el éxito académico no es tarea fácil, teniendo en cuenta que los estudiantes de posgrado no dedican a ello el $100 \%$ de su tiempo y que su disponibilidad es limitada, más todavía cuando el móvil que los lleva a cursar dichos programas es diverso y en la mayoría de las ocasiones se acompaña de responsabilidades laborales que los ocupan. Sin embargo, en atención a la responsabilidad social y el compromiso con la formación integral y de calidad que debe convocar a toda institución de educación superior, la retención estudiantil en posgrado debe cobrar relevancia y ocupar un lugar importante para la atención de los profesionales que vuelven al espacio universitario.

Que las universidades dispongan de redes para el estudio de la temática y para la colaboración en los procesos de retención en sus programas, facilita y optimiza los esfuerzos que desde diferentes espacios se llevan a cabo para apuntarle a la cobertura, la persistencia y la graduación, que para el caso de la educación posgradual redunda en beneficios de rendimiento o producción y calidad en los escenarios laborales.

Facilitar espacios y estrategias que de manera flexible permitan a los estudian-

tes fortalecer los aspectos académicos en los que se siente debilidad, es un llamado que en términos de formación deben acoger instituciones y programas como un aporte a la calidad de la educación posgradual en el país. 


\section{Referencias Bibliográficas}

AICD-Organización de Estados AmericanosOEA, Agencia Interamericana para la Cooperación y el Desarrollo AICD. (2006). Estrategias y materiales pedagógicos para la retención escolar.

Attinasi, L. (1986). Getting in: Mexican American Students' perceptions of their collegegoing behavior with im $\neg$ plications for their freshman year persistence in the University. ASHE 1986 Annual Meeting Paper, ERIC № 268 869. San Antonio, TX.

Barragán, D. y Quiroga, L. (2004). Pensar lo humano en los estudios de posgrado en perspectiva de los derechos. Bogotá, Colombia: Universidad de La Salle.

Barrientos, Z. y Umaña, R. (2009). Deserción estudiantil en posgrados semipresenciales de la Universidad Estatal a Distancia (UNED). Cuadernos de Investigación UNED. V. 1 (2). $141-150$.

Cabrales, O. (2008). Contexto de la evaluación de los aprendizajes en la educación superior en Colombia: sugerencias y alternativas para su democratización. Revista Educación y Desarrollo Social, 2(1), 141-165.

Castaño, E., Gallón, S., Gómez, K. y Vásquez, J. (2004). Deserción estudiantil universitaria: una aplicación de modelos de duración. Lecturas de Economía, 60, 39-65.

CEPAL. (2010). Panorama social de América Latina. Comisión Económica para América Latina y el Caribe.

Consejo Nacional de Educación Superior - CESU. (2013). La voz de las regiones. Aportes de la sociedad civil y la comunidad académica en los Diálogos Regionales sobre Educación Superior, promovidos por el CESU entre marzo y junio de 2013. Recuperado de http:// www.dialogoeducacionsuperior.edu.co/1750/articles-326326_archivo_pdf_lavozdelasregiones.pdf

Esquilver, L. y Rojas, C. (2002). Motivos de estudiantes de nuevo ingreso para estudiar un posgrado en educación. Yucatán, México: Universidad Autónoma de Yucatán.

Ethington, C. (1991). A psychological model of student persistence. Research in Higher Education. №31, Vol. 31: 279-293.

Fernández, N. (2009). Retención y persistencia estudiantil en instituciones de educación superior. Caracas, Venezuela: Universidad Simón Bolívar.

Fishbein, M. y Ajzen, I. (1975). Betief, atutude, intention, and behauior: An introduction to theory and research. Reading, MA.: Addison, Wesley.

Flores, A. (2008). La vida académica y su relación con la eficiencia terminal en los programas de posgrado de Sociología y Ciencia Política de la Facultad de Ciencias Políticas de la UNAM. México D.F., México: CIIEA. 
García, A. y Navarrete, M. (s. f.). Estrategias para mejorar la calidad educativa, con énfasis en la retención y eficiencia terminal. Recuperado de http://www.alfaguia.org/alfaguia/ files/1320781245_9317.pdf

Georgina, N. (2010). Valores universitarios y profesionales de los estudiantes de posgrado de la UNAM.RevistadelaEducación Superior, 39(154), 139-145. Recuperadodehttp://www. scielo.org.mx/scielo.php?script=sci_arttext\&pid=S0185-27602010000200008\&lng $=e s \&$ tlng $=$ es

Giogvagnoli, P. (2001). Determinantes de la deserción y graduación universitaria. Documento de trabajo n. ${ }^{\circ}$ 37. La Plata, Argentina: Universidad Nacional de la Plata.

Guzmán, P. (2009). Deserción y retención estudiantil en los programas de pregrado de la Pontificia Universidad Javeriana. Bogotá, Colombia: Pontificia Universidad Javeriana.

Guzmán Ruiz, C., Durán Muriel, D., Franco Gallego, J., Castaño Vélez, E., Gallón Gómez, S., Gómez Portilla, K. y Vásquez Velásquez, J. (2009). Deserción estudiantil en la educación superior colombiana: metodología de seguimiento, diagnóstico y elementos para su prevención. Bogotá, Colombia: MEN.

lesalc, Informe sobre la Educación Superior en América Latina y el Caribe. (2006). The Task Force on Higher Education and Society, The World Bank, 2000, Statistical Appendix; Informes Nacionales.

Martínez, G., Urrutia, A., Ponces, R. y Gil, M. (2003). Perfil del estudiante de posgrado con éxito académico en la UNAM. Tarbiya, 32, 133-145.

McFadden, M. y Munns, G. (2002). Student engagement and the social relations of pedagogy. British Journal of Sociology of Education, 23.

Ministerio de Educación Nacional - MEN. (2008). Deserción estudiantil en la educación superior colombiana. Elementos para su diagnóstico y tratamiento. Recuperado de http://www.mineducacion.gov.co/sistemasdeinformacion/1735/articles-254702_diagnostico_desercion.pdf

Misas, A. (2004). La educación superior en Colombia: análisis y estrategias para el desarrollo. Bogotá, Colombia: Universidad Nacional de Colombia.

Morales, A., Nolasco, S. y Anzaldo, E. (2003). Vida académica y eficiencia terminal del nivel superior de la Universidad Autónoma de Nayarit 1995-2001: una mirada retrospectiva en el marco del modelo educativo tradicional. Ponencia presentada en el $X$ Congreso Internacional Retos y Expectativas de la Universidad, en la Universidad Autónoma de Nayarit, México.

Ochoa, L. y Cueva A. (2012). Tesis y deserción: entre el compromiso y el obstáculo. Bogotá, Colombia: Universidad Nacional de Colombia. 
Páramo, G. y Correa, C. (2012). Deserción estudiantil universitaria. Conceptualización. Revista Universidad EAFIT, 35(114), 65-78. Recuperado de <http://publicaciones.eafit. edu.co/index.php/revista-universidad-eafit/article/view/1075/967>.

Pineda, C. y Pedraza, O. (2011). Persistencia y graduación: hacia un modelo de retención estudiantil para instituciones de educación superior. Bogotá, Colombia: Colciencias/ Universidad de La Sabana.

Rivera Rearte, E. A., Roca Soruco, H. N., Echart Limachi, B., Alfaro Murillo, E., López Reinoso, A. R., Farfán Sossa, S., Mercado, C., et al. (2005). Estudio sobre repitencia y deserción en la educación superior en Bolivia. Tarija, Bolivia: Universidad Autónoma "Juan Misael Saracho". Recuperado de http:/www.alfaguia.org/alfaguia/ files/1318974304Estudio\%20sobre\%20repitencia\%20y\%20desercion\%20en\%20 la\%20educacion\%20superior\%20en\%20Bolivia.pdf

Rizzuto. F (2009). La deserción de la educación superior, motivos y medidas preventivas. Valdivia, Chile: Universidad Austral. Recuperado de http://web.austral.edu.ar/descargas/escuela-educacion/tesis-Flavia-Rizzuto.pdf

Salcedo, A. (2010). Deserción universitaria en Colombia. Revista Academia y Virtualidad, 3(1), 50-60.

Sánchez, R. (2007). La teoría de los campos de Bourdieu, como esquema teórico de análisis del proceso de graduación en posgrado. Revista Electrónica de Investigación Educativa, 9(1).

. (2009). ¿Quiénes son los estudiantes de la Maestría en Pedagogía de la UNAM?: influencia del capital cultural y el habitus en el desarrollo académico en un posgrado. Revista electrónica de investigación educativa, 11(1), 1-22. Recuperado de http://www. scielo.org.mx/scielo.php?script=sci_arttext\&pid=\$1607-40412009000100005\&lng =es\&tlng=es

Sánchez, F., Márquez, J. (2012). La deserción en la educación superior en Colombia durante la primera década del siglo XXI: ¿Por qué ha aumentado tanto? Bogotá, Colombia: Universidad de Los Andes.

Sisto Campos, V. (2005). Flexibilización laboral de la docencia universitaria y la gestación de la universidad sin órganos. Un análisis desde la subjetividad laboral del docente en condiciones de precariedad. En: Gentili, P. y Levy, B. (comp.). Espacio público y privatización del conocimiento. Buenos Aires, Argentina: Clacso. Recuperado de: http:// bibliotecavirtual.clacso.org.ar/ar/libros/lbecas/espacio/Campos.pdf

Torres, L. (2010). Estado del arte de la retención de estudiantes de la educación superior. Bogotá, Colombia: Pontificia Universidad Javeriana. 
Universidad Nacional de Colombia. (2011). La deserción estudiantil de la Universidad Nacional de Colombia en el sistema de educación superior. Bogotá, Colombia: Autor. Unesco, Oficina Regional de Educación para América Latina y el Caribe. (2012). Situación educativa de América Latina y el Caribe. Hacia una educación para todos 2015. México D.F., México: Secretaría de Educación Privada. Recuperado de http://www. unesco.org/new/fileadmin/MULTIMEDIA/FIELD/Santiago/pdf/situacion-educativa-mexico-2013.pdf

Vásquez, J., Castaño, E., Gallón, S. y Gómez, K. (2003). Determinantes de la deserción estudiantil en la Universidad de Antioquia. Medellín, Colombia: Universidad de Antioquia.

Zabalza, M. (2007). La enseñanza universitaria, el escenario y sus protagonistas. Madrid, España: Narcea. 